Artículo de reflexión

Cómo citar: Gomez-Barrera, J. (2021). Estudiar las redes sociales: perspectivas teóricas para un análisis, Mediaciones, 26 (17) Pp. 190-203. https://doi.org/10.26620/uniminuto. mediaciones.17.26.2021.190-203

Editorial: Corporación Universitaria Minuto de Dios - UNIMINUTO

Recibido: 27 de abril de 2021 Aceptado: 28 de abril de 2021 Publicado: 23 de junio de 2021

ISSN: 1692-5688 | eISSN: 2590-8057

Juan Camilo Gómez-Barrera jcgomezbalgmail.com

Magíster en Comunicación y Cultura Facultad de Ciencias Sociales Universidad de Buenos Aires Colombia

\section{Estudiar las redes sociales: perspectivas teóricas para un análisis}

\section{Studying social media networks: theoretical perspectives for an analysis}

\section{Estudo das Redes Sociais: Perspectivas Teóricas para uma Análise}

Juan Camilo Gómez-Barrera

\section{Resumen}

Las actuales redes sociales digitales se han convertido en un foco de múltiples análisis y temas de investigación. Como consecuencia, circulan en las diversas bases de datos múltiples investigaciones que privilegian marcos conceptuales y referentes teóricos sobre ciertos temas de interés en el análisis de las redes sociales. Frente a esto, el objetivo del presente artículo es mostrar dos marcos conceptuales diferentes desde los cuales se pueden analizar las redes sociales, que se distancian de las miradas, los temas y los marcos teóricos privilegiados sobre el estudio de las redes sociales más abundantes, y que a su vez permiten comprender su relación con las máquinas y los problemas socio-políticos. Con ello, se pretende aportar elementos teóricos a investigaciones que giren en torno a preguntas como ¿qué son las redes sociales y cómo se las puede estudiar en torno a problemas socio-políticos?

Palabras claves: Redes sociales, teorías, Simondon, gubernamentalidad algorítmica

\begin{abstract}
Currently, the online social networks have been studied by diverse studies and research. As a consequence, there is a lot of research in data bases that highligts analytical interest in social media through certain specific conceptual frameworks and theoretical referents. As a result, this paper aims to show two specific frameworks that allow to understand and to study social networks around the problem of machines and their relationship with social and political problems. These frameworks, hence, allow to analyze other problems beyond the main problems and topics that are common in the studies that appear in specialized journals. In sum, the aim is to expose two frameworks that allow to answer questions like: What is an online social network? And what is its relation to the social and political spheres?
\end{abstract}

Keywords: conline social networks, frameworks, Simondon, algorithmic governmentality. 
Atualmente, as redes sociais online têm sido estudadas por diversos estudos e pesquisas. Como conseqüência, existem muitas pesquisas nas bases de dados baseadas em estruturas específicas e principais em

detrimento de outras. Como resultado, este artigo tem como objetivo mostrar duas estruturas específicas que permitem compreender e estudar as redes sociais em torno do problema da máquina e sua relação com problemas sociais e políticos. Essas estruturas, portanto, disponibilizam a análise de outros problemas além dos principais problemas e tópicos comuns nos estudos que aparecem em periódicos especializados. Em resumo, o objetivo é expor duas estruturas que permitem responder a perguntas como: O que é uma rede social online? E, qual é a relação com os panoramas sociais e políticos?

Palavras-chave: Redes socias, referencial teórico, Simondon, guvermentalidad algoritmica.

\section{Introducción}

Las redes sociales, entendidas como plataformas que permiten compartir información, entablar relaciones con diversos usuarios y ver información de ellos (Boyd y Ellison, 2007), se han convertido en parte indispensable de las relaciones sociales de los individuos. Principalmente, esto se debe a los cambios en las formas de adquirir información, en la manera como se ejercen escenarios políticos, negocios e incluso los modos de vida (Dwivedi, Kapoor, y Chen, 2018; Kapoor et al., 2018; Ngai, Tao, y Moon, 2015, y Rathore, Ilavarasan, y Dwivedi, 2016). Todas estas nuevas prácticas han desarrollado múltiples enfoques de estudio y una producción casi inabarcable de análisis enfocados en diversas perspectivas. Algunos de los focos más analizados son, por ejemplo, el comportamiento de los usuarios (Lönnqvist y große Deters, 2016) y sus interacciones (Sagioglou y Greitemeyer, 2014), consecuencias directas en las formas en que se perciben los individuos (Brailovskaia y Bierhoff, 2016) y, en especial, las consecuencias sociopolíticas que conllevan las afectaciones de la privacidad (Junior, Xavier, y Prates, 2014; Külcü \& Henkoğlu, 2014; Saeri, Ogilvie, La Macchia, Smith, \& Louis, 2014 y Tsay-Vogel, Shanahan, y Signorielli, 2016).

Este último tema, cabe indicar, ha sido uno de los que más ha encontrado eco en prensa y en grupos activistas en distintos países. Una muestra de ello son las diversas fundaciones de derechos de Internet ${ }^{1}$, que han sacado a la luz pública temas tan controversiales como la reciente Facebook Analytica. Cabe indicar que los análisis sobre las afectaciones sobre la privacidad, de acuerdo con Hagerty (2014), se basan en conceptos como los del hecho social, enraizados en las teorías de Emile Durkheim. En consecuencia, teniendo como base la existencia de un marco social compuesto por individuos y grupos codependientes de factores económicos e institucionales, la privacidad se articula como

1 La APC publicó en 2017 el reporte "Examinando los derechos y las libertades en Internet en América Latina”21 (ExLiLa), que recopila un análisis de varios países en torno al estado de las normativas a la protección de los derechos de Internet. Se puede consultar en: http://www.apc.org/sites/default/files/EXLILA\%20Informe\%20consolidado\%20de\%20investigaci\%c3\%b3n.pdf 
un espacio en el que los ciudadanos están libres de escrutinio ${ }^{2}$. A partir de las diversas conclusiones que señalan cómo las redes sociales ponen en riesgo la privacidad (a través de tecnologías como los cookies ${ }^{3}$ ) se han postulado análisis que procuran formas en las que se garantice la capacidad autónoma del control de información que reposa sobre los usuarios; es decir, se apela a una regulación de los datos.

Sin embargo, los marcos conceptuales que se han abordado para el estudio de las redes sociales son mucho más amplios y abarcan otros aspectos. De acuerdo con Ngai, Tao, y Moon (2015), las teorías más relevantes en las que se han basado los estudios de las redes sociales son el comportamiento individual, teorías sobre el comportamiento social y teorías sobre los medios de comunicación masiva. La primera de ellas es un modelo que se ha enfocado en explicar las afectaciones de las redes sociales al comportamiento individual de los usuarios. El segundo grupo está relacionado con el estudio del comportamiento social que se da en el uso colectivo de plataformas como Facebook o Twitter. Por último, el tercer grupo, estudios de mass media se enfocan en la influencia que pueden tener estas plataformas en el comportamiento de los individuos (Ngai, Tao, y Moon, 2015).

Estos marcos conceptuales han derivado en diversos temas que, si se quiere, se han convertido en tendencias de análisis y en los modos de comprender las redes sociales. De acuerdo con Shiau, Dwidevi y Lai (2018), a través de una revisión de las publicaciones más importantes a partir de 2015, la mayoría de las publicaciones sobre las redes sociales han girado principalmente en torno a seis temas. El primero de ellos es el análisis del comportamiento de los usuarios; el segundo, el impacto social de las redes en la sociedad; el tercero tiene que ver con modelos de evaluación teórica; el cuarto tema gira en torno a la influencia y usos de las redes sociales en universidades; el quinto consiste en los riesgos a la privacidad y las interacciones interpersonales; y, por último, el estudio de las estrategias de las redes sociales.

Tanto los marcos conceptuales como las temáticas anteriores se sustentan en la categoría de uso de las redes sociales (Shiau, Dwidevi y Lai, 2018); es decir, parten de la comprensión y estudio de los modos en los que los usuarios se relacionan y entablan conexiones, tanto en su cotidianidad como en el interior de las plataformas. Este marco de comprensión, que proviene en especial de una crítica norteamericana, sin embargo, deja de lado otras formas de comprensión que no surgen del análisis de los modos de uso de las redes sociales. Una consecuencia inmediata de esto es que se limitan puntos de comprensión de elementos como las posibles relaciones entre mecanismos de poder y las redes sociales, o las formas y métodos de encarar el estudio de las redes sociales.

2 Un análisis más completo exigiría revisar los libros de Daniel Solove, en especial (2008). Understanding Privacy.

3 Un estudio introductorio al problema puede consultarse en: Monteiro, L. (2015). Vigilância e políticas de privacidade na sociedade pós-cookies: O caso do The Guardian. En: Revista Eco Pós. 18(2), págs. 95-105. 
No obstante, atender a otros marcos interpretativos precisa desplazar la relación de dependencia de las redes sociales con los usuarios; en otras palabras, exige pensar a las redes sociales por fuera de su relación inmediata con los individuos y, en contravía, analizarlas como objetos en sí, independientes del uso que se les da. Con ello, se abren formas de análisis en las que pueden estudiarse mecanismos, tecnologías y funciones que, en sí mismas, implican consecuencias y hechos que son objetos de estudio. En ese desplazamiento, uno de estos elementos que pueden analizarse son las convergencias con mecanismos de poder o las modalidades en las que prácticas políticas se alimentan de los mecanismos y herramientas propias de las redes sociales.

En consecuencia, el presente artículo pretende plantear dos marcos interpretativos sobre las redes sociales, paralelos a los predominantes en diversas publicaciones. El primero de ellos está centrado en las reflexiones sobre la filosofía de la técnica que pueden extraerse de las reflexiones de Gilbert Simondon sobre el análisis de los objetos técnicos en relación con la cultura. Con este marco se pretende generar metodologías de análisis que privilegien un estudio de las redes sociales en sí, independiente de conceptualizaciones enmarcadas en los individuos y que a su vez permitan pensar de otro modo las relaciones entre los sujetos y los complejos tecnológicos que los rodean. Esto permite analizar herramientas, funciones, mecanismos, etc., propios de las plataformas de las redes sociales que en sí generan consecuencias y puntos clave de interpretación.

El segundo marco, centrado en las reflexiones de Berns y Ruvroy (2016), busca encontrar los posibles vínculos, y modos de hallarlos, entre las redes sociales y prácticas políticas enmarcadas dentro de la noción de gubernamentalidad. Con ello se plantean formas de análisis que privilegien hipótesis relacionadas sobre las plataformas de Internet y mecanismos de poder; por tanto, se sugieren análisis encaminados a plantear las consecuencias políticas y sociales, desde una teoría del poder de las redes sociales.

No sobra indicar que estos dos marcos teóricos de referencia no son unívocos ni pretenden establecer jerarquías sobre los mencionados en un principio. Por el contrario, se pueden leer como bases teóricas que permiten analizar ciertos fenómenos de las redes sociales y, por otro lado, ayudan a develar nuevos procesos y objetos de estudio. En esa medida, deben considerarse como herramientas de análisis en las que predomina una lectura particular y específica, que pueden controvertirse, ampliarse o rechazarse.

\section{Primer marco. Las redes sociales como máquinas: una apuesta simondoniana}

Uno de los puntos centrales en un desplazamiento de los modos de análisis de las redes sociales puede pensarse a través de un estudio enfocado directamente en el objeto técnico. En esa medida, antes que deducir consecuencias sociales, políticas, económicas, etc., sobre las plataformas de Internet, es necesario considerarlas como objetos técnicos en sí, aptos de ser analizados y comprendidos internamente, independiente de miradas o factores externos. Como se dijo en la introducción, el eje de la mayoría de los análisis sobre el tema en cuestión está centrado en el problema del uso de las redes sociales, y desplazan aspectos analíticos de cómo se conforman, funcionan, estructuran y delimitan 
las plataformas. Desplazar este enfoque requiere centrarnos en un punto de articulación distinto que, a su vez, permita derivar múltiples temas, hipótesis, teorías y vínculos interpretativos con diferentes campos de conocimiento.

Un marco conceptual que nos resulta interesante para un propósito tal es el abordado por el filósofo francés Gilbert Simondon (1924-1989), quien, valga decirlo, ha sido uno de los descubrimientos teóricos más importantes dentro de la filosofía de la técnica del siglo $\mathrm{XX}^{4}$. Uno de los aspectos centrales en su pensamiento sobre la técnica tiene que ver con el hecho de que la tecnología tiene en sí misma un desarrollo autónomo. Es decir, parte de una unidad de análisis constituida por el objeto técnico y no tanto en cómo se lo ha leído o sus efectos y relaciones en la sociedad. De esta manera, el objeto técnico tiene una historia propia, interna, que se lleva a cabo a través de etapas; el proceso de este desarrollo se conoce como concretización. Esta postura centrada en el objeto técnico no determina que en el desarrollo tecnológico haya una intención humana. No obstante, el punto central para Simondon (2008) tiene que ver con que ambas relaciones y procesos de desarrollo, el humano y el de las máquinas, se articulan y se diferencian por distintos factores.

El concepto de concretización alude a la evolución de un objeto técnico, de acuerdo con unas pautas internas de tipos funcionales, estructurales y energéticos. Esto significa que es necesario restituirles a los aparatos técnicos una cierta autonomía ontológica, en donde resulte fundamental el estudio de la convergencia estructural y funcional de los componentes de cualquier artefacto. Así, es necesario entender las partes, las piezas, el modo en que se articulan, etc., para entender su desarrollo. En el caso específico de las redes sociales, y de acuerdo con esto, sería necesario entender aquellos elementos, procesos internos, partes y funciones que le son propias.

Siguiendo este principio teórico, se pueden distinguir dos componentes del proceso de concretización. Por un lado, la sobredeterminación, que se da siempre al interior del aparato técnico y que se puede comprender como el conjunto de las múltiples causas que determinan el funcionamiento de los componentes que constituyen un objeto técnico. Para ofrecer un ejemplo, vendría a ser la forma en la que se articulan los componentes de una red social; a saber, cómo se sobredeterminan funciones específicas de la plataforma como lo pueden ser la posibilidad de comentar, de manifestar el acuerdo o desagrado, las funciones de colgar una foto, entre otras. Por el otro lado, la indeterminación con la que se relacionan y convergen los procesos y componentes de un aparato técnico. Esto teniendo en cuenta que, en un objeto técnico concreto, todas las partes son "integradas"; esto es, que la "esencia de la concretización del objeto técnico es la organización de subconjuntos funcionales en el funcionamiento total” (Ibíd., p. 56). Para entenderlo mejor, es necesario analizar y establecer cómo ciertas estructuras y herramientas de una plataforma resultan funcionales entre sí: por ejemplo, bajo qué medida es necesario para una red social como Facebook la existencia del botón de comentar en un estado o en una publicación cualquiera.

4 Para un panorama general de los aportes y discusiones en torno a los aportes de Simondon sobre el estudio de la técnica, puede consultarse: Blanco, J. et al. (2015). Amar a las máquinas: cultura y técnica en Gilbert Simondon. Buenos Aires: Prometeo Libros. 
Por tanto, estas dos dimensiones de la concretización invitan a pensar que no se deben comprender elementos o partes aisladas de un objeto técnico y, al reducirlas, determinar hipótesis interpretativas de este. Por el contrario, se deben articular todas en conjunto, pues son las que pertenecen, como tal, a un objeto técnico.

La pregunta por la concretización de los objetos técnicos remite, en esa medida, a una pregunta por la génesis de estos aparatos 5 . Para Simondon (2007), los objetos técnicos comportan una similitud con los seres biológicos por un principio de herencia genética de sus antecesores. El desarrollo de una plataforma de Internet tiene unas raíces en otro tipo de aparatos y plataformas que en su mayoría comparten funciones, herramientas, sistemas. En otras palabras, la concretización nos permite entender que el proceso de desarrollo de los aparatos técnicos tiene una trayectoria histórica que muchas veces sigue evolucionando. Por tanto, un posible estudio de las redes sociales debería también partir de comprender cómo se relacionan y qué elementos extrae de aparatos o tecnologías anteriores. Con ello, podrían ponerse en discusión, por ejemplo, hasta qué punto las prácticas que se consideran desplegadas con el surgimiento de las redes sociales derivan de usos y tecnologías anteriores: el hecho de comentar, si se quiere, puede derivar en un análisis de cómo ciertas tecnologías previas determinaron rasgos técnicos a otros tipos de medios que propiciaron dinámicas similares, como lo pudo ser la prensa o la constitución de una opinión pública.

Así mismo, esta derivación genética de los objetos técnicos debe partir, de acuerdo con Simondon (2008), del estudio de una esencia técnica que contiene ya un horizonte de posibilidades. Esta esencia no es solo una forma de entender el objeto técnico, sino que se trata de un esquema de funcionamiento que permite determinar aquello que cierto objeto técnico puede efectuar. En el caso de las redes sociales, sería necesario establecer históricamente aquello que les es propio, los elementos que fueron funcionales y que determinaron su desarrollo posterior. Por ejemplo, sería necesario estudiar los elementos originales de una red social como Twitter, sus funciones y herramientas, y cómo estas determinaron el desarrollo de su plataforma, de sus funciones y de sus posibilidades. Allí debería estudiarse el sentido que pudo tener el uso limitado de palabras para las publicaciones que, más allá de ser un anclaje técnico, determina el uso que se le pueda dar a la plataforma y las consecuencias (lingüísticas, políticas, educativas) que se quieran desarrollar.

Visto esto, concretización y génesis son conceptos que se articulan en Simondon (2007). De la comprensión de estos dos se puede entender que un aparato técnico se comporta como un individuo, entendido como aquel que es el resultado de una "fijación material" de un proceso o un "gesto", que lo hace para sí mismo a través de una invención (Parente y Sandrone, 2015). Es decir, esto también puede entenderse bajo la pregunta de aquella función técnica que permitió constituir el aparato técnico, que delineó y le dio sus funciones actuales. Esto resulta muy interesante para el estudio de las redes sociales

5 Cabe decir que el concepto de genealogía se acuña y pone en circulación con una tradición que se ancla en la genealogía nietzscheana, a la par de los desarrollos teóricos sobre la genealogía que desarrolló Michel Foucault. Para ello, puede consultarse el artículo escrito por Foucault, "Nietzsche, la genealogía, la historia”, publicado en diversas compilaciones. 
porque exige que su estudio deba partir de las causas, metodológicas, técnicas, etc., que impriman o forjen su aparición. Por ejemplo, sería necesario entender las funciones que resultan más centrales en una plataforma, anclado al desarrollo y evolución interna.

En esa instancia, una primera invitación para el estudio de las redes sociales está enfocado en analizar sus partes técnicas, su origen, sus características, etc. En esa medida, es necesario no solo indicar que las redes sociales, por ejemplo, afectan los modos en los que se relacionan los individuos, sino que habría que complementar, indicando qué mecanismos internos en las redes sociales permiten efectuar esto. Esto es, analizar cómo se implementó la función de subir una foto, cómo se abrió la posibilidad de generar comentarios y qué objetivos, proyectos, mecanismos estuvieron en el fondo de estas funciones. En esa medida, se podrían comprender, dado el caso, los protocolos, las interfaces, las conexiones y mecanismos algorítmicos que subyacen; en gran medida, este análisis permite comprender las nuevas formas en que funcionan las redes sociales, y descentrar una mirada neutral de su modo de almacenamiento de información.

\section{Segundo marco. Redes sociales y el poder: los aportes de la gubernamentalidad algorítmica.}

Una de las consecuencias que pueden determinarse del estudio del objeto técnico en sí es que muchos de sus mecanismos están centrados en funciones algorítmicas. El hecho de comentar algo, de colgar una foto, de agregar a la lista de amigos a un perfil determinado, etc., están anclados a funciones que se desenvuelven en un terreno específicamente matemático. De por sí, esta conclusión no resulta del todo obvia y, por el contrario, permite comprender muchos fenómenos que se pueden derivar de este funcionamiento. Uno de ellos tiene que ver con la incidencia que pueden llegar a tener los algoritmos en ámbitos políticos y sociales. Una pregunta que puede guiar un estudio relacionado puede ser ¿qué efectos conlleva el hecho de que un algoritmo sea aquello que determine, por ejemplo, a quién se debe seguir o qué tipo de contenido es el que un usuario debe ver? Para poder responder las consecuencias posibles de tal pregunta, consideramos que un campo de estudio sobre el que se pueden sustentar muchas de ellas es el de la gubernamentalidad algorítmica.

Es Antoinette Rouvroy quien acuñó el concepto de "gubernamentalidad algorítmica" a partir del trabajo sobre los modos de ejercicio de poder en la biopolítica, desarrollado por Foucault en los cursos en el Collège de France entre 1976-1980. Con este concepto quiere dar a entender la existencia de un gobierno estadístico que es capaz de rastrear, de manera readaptable, a toda la población. Los estudios sobre gubernamentalidad actuales pretenden cartografiar la relación entre las racionalidades y las tecnologías usadas para gobernar conductas, comportamientos, acciones. Para ello, se buscan contestar preguntas en torno a las tecnologías que permiten producir procesos de subjetivación y mecanismos de poder. De igual modo, se interroga por los modos en los que se estructura un gobierno de lo social, en el cual se puede entender el papel del Estado y la economía como entidades reguladoras y centralizadas. En esa medida, los 
temas que abarca esta propuesta teórica son mucho más amplios y complejos que temas relacionados con la privacidad, en lo referente al estudio de la tecnología.

De forma análoga, se piensan dentro de esta teoría autores diversos como Eric Sadin, quien resalta en la escena francesa; los algorthimic studies, que incluyen a autores como el norteamericano Ted Striphas; o la noción de perfilización de la vida, de la brasileña Fernanda Bruno. En esa instancia, se interconectan temas como la medicalización de la población, estudios sobre el control de poblaciones a través de los medios de comunicación y las formas de almacenamiento de datos. A su vez, y en contacto con teorías sobre el neoliberalismo, también se abarcan campos como la interpelación de ciudadanos emprendedores, la constitución de sujetos económicos autogestionados, etc.

En esa medida, para el caso particular, las redes sociales se entienden como uno de los actores que recopilan grandes masas de datos, funcionales para instituciones y Gobiernos de turno. Todo ese andamiaje y proceso de recopilación, venta y uso de datos con fines de gobierno están inmersos en una lógica, o una racionalidad, que pone en el centro el uso de algoritmos. Efectivamente, no se pueden pensar las redes sociales por fuera de elementos sustanciales de funcionamiento como estas operaciones matemáticas, que le dan forma y les permiten ser aprovechadas por mecanismos y aparatos de vigilancia.

De esa manera, la gubernamentalidad algorítmica la podemos entender como "cierto tipo de racionalidad (a)normativa o (a)política que reposa sobre una recolección, agregación y análisis automatizada de datos en cantidades masivas de modo que se pueda modelizar, anticipar y afectar, por anticipación, los comportamientos posibles” (Rouvroy y Berns, 2016, p. 41). Es decir, es una forma de ejercer el gobierno a partir de los datos, que tiene un anclaje muy inmediato en la estadística. Si bien desde el siglo XIX se inició un gobierno que tenía como base el registro de datos sobre el estado de cosas de un país, la característica fundamental del modo de operación de la gubernamentalidad actual es la centralidad que tienen los algoritmos.

Así, pues, esta extracción automatizada de datos "pertinentes” de usuarios cuya finalidad incluye actores políticos y mercantiles, tiene para Ruvroy y Berns (2016) por lo menos dos consecuencias, o si se quiere, efectos sobre la sociedad. La primera de ellas consiste en la creación de una dupla de lo real. Este procedimiento consiste en una serie de operaciones que se inician con una recolección de datos, cuyo ejemplo se ve en la existencia de los datawarehouses, elementos constitutivos de la denominada Big data. Esta recolección de datos no es para nada novedosa en las redes sociales actuales y, sí, por el contrario, constituye una de las funciones básicas de operación de estas. El tema central para los autores consiste no tanto en el hecho de extracción de los datos, sino que estos son utilizados como elementos que han sido vaciados de su contexto y con ello se ven reducidos a su "naturaleza más bruta". De esta manera, estos datos son trabajados por las distintas entidades como una materia absolutamente objetiva sobre la realidad, que no deja de ser problemática. Por ejemplo, si por error se ha oprimido algún botón en una red social, esto se convierte en un dato que permite inferir múltiples conclusiones más allá de las razones que un usuario pudo llegar a tener al momento de efectuarlo. 
Todo el trabajo de extracción y almacenamiento sobre información de los usuarios, tema que ha sido abordado por múltiples análisis, es producto de programas que son capaces de reconocer emociones y comportamientos implícitos en la información, y transformarlos en un plano estadístico. Así, se establecen datos para determinar cuántas veces visitó cierto perfil en una red social, cuántas veces escribió cierta palabra, etc. Esta recolección de datos no solo tiene una finalidad comercial -como calcular las probabilidades de venta de un producto o su receptividad-, sino que permite realizar ejercicios de gubernamentalidad como delimitar muestras de intenciones de voto, tendencias ideológicas, etc.

Además de la operación de recolección de datos, cuyas consecuencias son poner como eje central datos objetivos que vienen a dar cuenta de la realidad, Ruvroy y Berns (2016) indican que hay una operación de procesamiento y análisis de dichos datos, denominada datamining, o minería de datos, cuyo objetivo más relevante es efectuar relaciones entre los datos. La búsqueda de conexiones, coincidencias, rupturas, en fin, toda la operación de correlación de los datos almacenados a partir de su procesamiento tiene la función de "producir un saber" estadístico que se elabora a partir de las correlaciones de informaciones heterogéneas. Lo fundamental de esta operación es que esta producción de saber, que podía encontrarse antes en ciencias como la medicina, ciencias judiciales, pedagógicas, es ahora automatizado por medio de algoritmos; es decir, es un saber producido con una intervención mínima de la mirada humana y, como lo indicamos anteriormente, evitando cualquier forma de subjetividad.

Tenemos, en consecuencia, en un primer nivel de análisis de Ruvroy y Berns (2016), el manejo de los datos que se realizan en la gubernamentalidad algorítmica. En este nivel, encontramos una extracción y almacenamiento, procesamiento y producción de saber, y un actuar sobre estos datos extraídos; que no son otra cosa que las huellas de los usuarios, sus comportamientos puestos en datos y estadísticas. Sin embargo, los efectos de este trabajo sobre los datos con operaciones automatizadas van mucho más allá de este primer nivel.

La segunda consecuencia que señalan Ruvroy y Berns (2016) tiene que ver con el hecho de que, al ser un algoritmo el que realiza las operaciones de análisis y de extracción, se establece una especie de "gobierno sin sujeto". El trabajo sobre los datos encuentra y evita a los humanos; al alimentarse de datos "infraindividuales", que aisladamente son insignificantes, crea perfiles de comportamientos que son “supraindividuales”, que van más allá de los individuos concretos, y bajo esa operación evita el contacto o interpelación directa a cualquier sujeto: "pero sin jamás convocarlo o darse cuenta por sí mismo de aquello que es, ni aquello en lo que podría convertirse" (Rouvroy y Berns, 2015, p. 40). Para volver al ejemplo de la red social, un clic sobre cierta foto, que puede ser accidental para el usuario, se convierte en un gesto diciente y determinante para el individuo. Lo mismo puede suceder en la escritura de ciertas palabras que, ya sea por error, son determinadas como "peligrosas" o "problemáticas" para los algoritmos. Este posible señalamiento sobre un individuo se torna mucho más diciente e importante (para el comercio o sistemas de vigilancia) que el sujeto de carne y hueso, dicho en forma coloquial. Sus datos, el análisis efectuado sobre estos, 
las relaciones digitales y su comportamiento en Internet dibujan a un individuo que reemplaza al original, aunque las consecuencias, que dependen de la gravedad de sus fines, siempre recaigan sobre el humano.

No quiere decir esto que exista una supresión de los sujetos, como entidades concretas individuales. Por el contrario, señalan Ruvroy y Berns (2016) que hay un surgimiento de "hipersujetos" que adquieren forma únicamente como potencia; es decir, como producción de sujetos que están centrados no tanto en lo que son, o en el presente que los atraviesa, sino en lo que van a hacer en su futuro; esto es, el gobierno algorítmico se enfoca en las potencias de los sujetos, en sus acciones a largo plazo, en lo que pueden comprar, en lo que pueden efectuar, pues es justamente esta la finalidad que se encuentra en el saber en torno a, por ejemplo, qué tipo de producto podría gustarle a un usuario específico o qué tipo de ideología comporta cierta población en el mundo.

En síntesis, la supresión de los sujetos como objeto de acción directa de la gubernamentalidad implica un trabajo directo sobre las relaciones que se ponen en juego tras la extracción de información. Ruvroy y Berns (2016) entienden los datos como puras relaciones, o mejor dicho, cruces de informaciones; los saberes que se producen son "relaciones" de las "relaciones", y las acciones normativas se aplicarían al producto de los saberes que arrojan los entrecruces de datos. De esa manera, la gubernamentalidad algorítmica se centra en un trabajo en el que los seres humanos se tornan invisibles, pues solo se registran sus datos, y a la vez, aparece la potencia hacia el futuro, las probabilidades de acción, como el elemento central sobre el que se debe actuar. La consecuencia más inmediata es que los sujetos se tornan inoperantes en este nuevo tipo de ejercicio del poder, así como sus acciones y su tiempo presente se diluye en las acciones que probablemente haga en un futuro; cuyo ejemplo más determinante es la centralidad que tienen hoy día las compañías de seguro, determinantes de lo que se debe considerar como la vida.

Una clara muestra de cómo los gobiernos y las distintas instituciones de seguridad toman como eje de estudio todo el registro de datos en las redes sociales se puede observar en las distintas políticas de seguridad que ha asumido el Gobierno de los Estados Unidos. A partir de los sucesos del llamado "9/11", distintas modalidades de "identificación y prevención” han venido siendo incorporadas con más intensidad por parte de las distintas agencias de seguridad nacional. En este panorama, la información compartida por los usuarios en redes sociales ha devenido, con más intensidad, en un objeto de intervención y de estudio. De esta manera, los distintos planes de "intervención” en distintos agentes de la población, basados todo potencial peligro representativo, incluyen un seguimiento detallado de la función del aplicativo en cuestión, tal como lo han señalado en diversas ocasiones los informes del FBI, denominados "Homeland Security Committee".

Bajo esa perspectiva teórica, en conclusión, deben relacionarse las formas técnicas de funcionamiento de un aparato técnico, que pueden detenerse en la forma en la que extraen y almacenan datos, con consecuencias y políticas estatales sobre vigilancia y gobierno de las poblaciones. Esto es, los estudios sobre las redes sociales pueden enfocarse en el análisis de cómo la extracción de datos y modo de funcionamiento técnico permiten articular 
mecanismos de poder, como la vigilancia. Estos estudios, sobre los cuales ya se ha avanzado ${ }^{6}$, son necesarios porque los mecanismos y modos de funcionamiento de las redes sociales son cambiantes. Nuevas tecnologías aparecen y dan forma a nuevas formas de extracción de datos, a la par que suceden nuevas formas de determinación algorítmica de los sujetos.

\section{Conclusiones}

Las actuales redes sociales digitales se han convertido en el foco de múltiples análisis y temas de investigación. Circulan por Internet tesis, libros, artículos de investigación y notas de prensa en torno a cómo afectan, influyen e, incluso, ponen en riesgo nuestra vida social y privada. Algunos hechos que han determinado esto han surgido de conocidos casos como los de violaciones al acceso de datos o venta de estos a compañías con quienes los usuarios no han aceptado un contrato virtual.

Dentro de los diversos campos de estudio y enfoques teóricos que han surgido en torno a las redes sociales, encontramos el análisis del comportamiento de los usuarios, el impacto social de las redes en la sociedad, los denominados modelos de evaluación teórica, la influencia y usos de las redes sociales en universidades, los riesgos a la privacidad y las interacciones interpersonales y, por último, el estudio de las estrategias de mercado de las redes sociales.

Estos temas, sin embargo, pueden agruparse bajo la noción de uso que los usuarios depositan o practican en las plataformas. Se considera, en consecuencia, que es necesario ampliar cierto margen de comprensión e interpretación a través de marcos teóricos distintos, que permitan complejizar y problematizar las redes sociales.

El modo de efectuar estos nuevos análisis debe partir de nuevos marcos teóricos y referencias conceptuales. Curiosamente, muchos de estos desplazamientos posibles pueden venir de una teoría que no es del todo norteamericana. Así, hemos indicado dos corrientes europeas. Con estos referentes, consideramos que pueden emprenderse nuevas formas de estudio y análisis de las redes sociales, o de las plataformas de Internet, que estén enfocadas más en las lógicas que subsisten en su funcionamiento, en las racionalidades que implican, en los modos de operación o en las afectaciones y efectos sobre las subjetividades modernas.

En esa instancia, se reseñaron, a través de una mirada puramente panorámica sobre los cuerpos teóricos, dos teorías. Fueron reseñas que no tienen, valga decirlo, una pretensión de totalidad; por el contrario, existen allí otros conceptos que pueden ser enriquecedores para otros tipos de análisis. No obstante, consideramos que los mencionados son los más útiles para una primera aproximación.

6 Una buena fuente para recopilar y acceder a diversas investigaciones sobre el tema, desde Latinoamérica, es la red latinoamericana de estudios en vigilancia, tecnología y sociedad (Lavits). Han sido múltiples los coloquios, las investigaciones publicadas y los colaboradores que permiten rastrear fuentes y proyectos de investigación. Todo ello se puede consultar en: http://lavits.org/?lang=es 
La primera de las teorías es la desplegada por Simondon, para quien el estudio de la tecnología debe centrarse sobre todo en el objeto técnico en sí. De esta forma, se debe privilegiar un análisis enfocado en el estudio de los aparatos que permita comprenderlos para derivar de allí hipótesis o interpretaciones. Esto es muy importante tenerlo en cuenta como metodología de análisis, ya que es frecuente encontrar análisis que derivan hipótesis de ciertas tecnologías, pasando por alto el funcionamiento interno. La consecuencia más inmediata de este posible error metodológico es falsear cierta esencia técnica y forzar el objeto técnico a dar cuenta de interpretaciones que, quizá, no se derivan de su determinación técnica.

Un segundo marco teórico reseñado tiene que ver con el análisis de los efectos políticos que pueden derivarse de las redes sociales. Como lo mencionamos, y sobre lo cual habría que detallar más, una de las teorías dominantes sobre los efectos que pueden tener las redes sociales en un campo de estudio como el del poder giran en torno a la noción de privacidad. Frente a ello, consideramos que se deben analizar modos más sutiles y profundos de interpretación, como lo puede ser la noción de gubernamentalidad algorítmica. Para ello, referenciamos el trabajo de Ruvroy y Berns (2016), quienes plantean, en términos generales y entre otros elementos, que uno de los efectos del uso masivo de datos tiene que ver con la posibilidad de borrar a los sujetos del mapa de decisiones políticas y, por otro lado, construir lógicas decisivas y analíticas que sean fragmentarias.

Para finalizar, en consecuencia, consideramos que estos dos referentes teóricos permiten abrir nuevas posibilidades de análisis. No quiere decir, y vale la pena resaltarlo, que se traten de elementos unívocos; por el contrario, en combinación con otros, permiten construir nuevas hipótesis y marcos de interpretación. Esto, justamente, es lo que consideramos puede ser uno de los llamados a investigar: ante los desarrollos técnicos acelerados y la aparición constante de tecnologías, los marcos teóricos y los análisis de investigación sobre sus efectos, implicaciones, usos, etc., deben estar en constante apertura y creación.

\section{Referencias bibliográficas}

Berns, T., y Rouvroy, A. (2016). Gubernamentalidad algorítmica y perspectivas de emancipación. ¿La disparidad como condición de individuación a través de la relación? Adenda Filosófica, diciembre (1), pp. 88-116.

Boyd, D. M., \& Ellison, N. B. (2007). Social network sites: Definition, history, and scholarship. Journal of Computer-Mediated Communication, 13(1), pp. 210-230.

Brailovskaia, J., \& Bierhoff, H. W. (2016). Cross-cultural narcissism on Facebook: Relationship between self-presentation, social interaction and the open and covert narcissism on a social networking site in Germany and Russia. Computers in Human Behavior, 55 (A), pp 251-257

Debord, G. (1994). La sociedad del espectáculo. Ediciones naufragio. 
Deleuze, G. (1999), Posdata sobre las sociedades de control. En Christian Ferrer (Ed.), El lenguaje libertario. Antología del pensamiento anarquista contemporáneo. Altamira.

Dwivedi, Y. K., Kelly, G., Janssen, M., Rana, N. P., Slade, E. L., \& Clement, M. (2018). Social media: The good, the bad, and the ugly. Information Systems Frontiers, 20(3), 419-423.

Haggerty, K., (2014), What's Wrong with Privacy Protections? Provocations from a Fifth Columnist. En Sarat, A. A. (Ed.) World without Privacy: What Law Can and Should Do? Amherst.

Junior, M. P., Xavier, S. I. D. R., \& Prates, R. O. (2014). Investigating the use of a simulator to support users in anticipating impact of privacy settings in Facebook. Proceedings of the 18th International Conference on Supporting Group Work. ACM, pp 63-72.

Kapoor, K. K., Tamilmani, K., Rana, N. P., Patil, P., Dwivedi, Y. K., \& Nerur, S. (2018). Advances in social media research: Past, present and future. Information Systems Frontiers, 20(3), pp. 531-558.

Külcü, Ö., \& Henkoğlu, T. (2014). Privacy in social networks: An analysis of Facebook. International Journal of Information Management, 34(6), pp. 761-769

Lönnqvist, J. E., \& große Deters, F. (2016). Facebook friends, subjective well-being, social support, and personality. Computers in Human Behavior, 55(A), pp. 113-120.

Lucero Benavidez, Karla Lorena (2015). Proyección de la imagen fotográfica en Facebook: estudio de caso de un grupo de jóvenes desde una perspectiva de género. [Tesis de Maestría en Comunicación no publicada]. Universidad Andina Simón Bolívar, Ecuador.

Nash, A. (2016). Affect, People, and Digital Social Networks. En Tettegah, S. (Ed.). Emotions, Technology, and Social Media. Elseiver. pp 3-25.

Ngai, E. W. T., Tao, S. S. C., \& Moon, K. K. L. (2015). Social media research: Theories, constructs, and conceptual frameworks. International Journal of Information Management, 35, pp. 33-44.

Parente, D. y Sandrone, D., (2015), Invención y creatividad en la evolución de los objetos industriales: exploración de algunos problemas simondonianos. En Blanco, J., Parente, D. y Rodríguez, P. (coord.), Amar a las máquinas: cultura y técnica en Gilbert Simondon (pp. 277-300). Prometeo Libros.

Rathore, A. K., Ilavarasan, P. V., \& Dwivedi, Y. K. (2016). Social media content and product co-creation: An emerging paradigm. Journal of Enterprise Information Management, 29(1), pp. 7-18.

Saeri, A. K., Ogilvie, C., La Macchia, S. T., Smith, J. R., \& Louis, W. R. (2014). Predicting Facebook users' online privacy protection: Risk, trust, norm focus theory, and the theory of planned behavior. The Journal of Social Psychology, 154(4), pp. 352-369. 
Sagioglou, C., \& Greitemeyer, T. (2014). Facebook's emotional consequences: why Facebook causes a decrease in mood and why people still use it. Computers in Human Behavior, 35, pp. 359-363.

Shiau, W. L., Dwivedi, Y. K. y Lai, H. H. (2018). Examining the core knowledge on Facebook. International Journal of Information Management, 43, pp. 52-63.

Simondon, G. (2007), El modo de existencia de los objetos técnicos. Prometeo.

Sinanan, J. (2016). Social Media and Sorting Out Family Relationships. En Tettegah, S. (Ed.), Emotions, Technology, and Social Media (pp. 63- 89). Elseiver.

Strano, M. M. (2008). User descriptions and interpretations of self-presentation through Facebook profile images. Cyberpsychology: Journal of Psychosocial Research on Cyberspace, 2 (2), article 5.

Tsay-Vogel, M., Shanahan, J., \& Signorielli, N. (2016). Social media cultivating perceptions of privacy: A 5-year analysis of privacy attitudes and self-disclosure behaviors among Facebook users. New Media \& Society, 20, (1), pp. 141- 161. 